\title{
Assessment of the anterior loop of the mandibular canal: A study using cone-beam computed tomography
}

\author{
Eduarda Helena Leandro do Nascimento ${ }^{1}$, Maria Luiza dos Anjos Pontual², Andréa dos Anjos Pontual ${ }^{2}$, \\ Danyel Elias da Cruz Perez² ${ }^{2}$ José Natal Figueiroa ${ }^{3}$, Marco Antônio Gomes Frazão ${ }^{4}$, \\ Flávia Maria de Moraes Ramos-Perez ${ }^{2, *}$ \\ ${ }^{1}$ Department of Oral Diagnosis, Division of Oral Radiology, Piracicaba Dental School, University of Campinas (UNICAMP), São Paulo, Brazil \\ ${ }^{2}$ Department of Clinical and Preventive Dentistry, School of Dentistry, Federal University of Pernambuco (UFPE), Recife, Pernambuco, Brazil \\ ${ }^{3}$ Instituto de Medicina Integral Professor Fernando Figueira - IMIP, Recife, Pernambuco, Brazil \\ ${ }^{4}$ Division of Oral Radiology, Recife Dental School (FOR), Recife, Pernambuco, Brazil
}

\section{ABSTRACT}

Purpose: Sufficient area in the interforaminal region is required for dental implant placement, and the anterior loop of the mandibular canal is located within the limits of this area. The aim of this study was to evaluate the prevalence and extent of the anterior loop in a Brazilian sample population using cone-beam computed tomography (CBCT).

Materials and Methods: CBCT images from 250 patients (500 hemimandibles) obtained for various clinical indications were randomly selected and evaluated to determine the presence and length of the anterior loop. The length of the anterior loop was then compared based on gender, age, and the side of the mandible. The data were analyzed using the Pearson chi-square test and linear regression analysis.

Results: An anterior loop was identified in $41.6 \%$ of the cases, and its length ranged from $0.25 \mathrm{~mm}$ to $4.00 \mathrm{~mm}$ (mean, $1.1 \pm 0.8 \mathrm{~mm}$ ). The loop had a greater mean length and was significantly more prevalent in males $(p=0.014)$. No significant differences were found between the right and left sides regarding length $(p=0.696)$ or prevalence $(\mathrm{p}=0.650)$.

Conclusion: In this study, a high prevalence of the anterior loop of the mandibular canal was found, and although its length varied greatly, in most cases it was less than $1 \mathrm{~mm}$ long. Although this is a prevalent anatomical variation, safety limits for the placement of implants in this region cannot be established before an accurate evaluation using imaging techniques in order to identify and preserve the neurovascular bundles. (Imaging Sci Dent 2016; 46: 69-75)

KEY WORDS: Anatomic Variation; Cone-Beam Computed Tomography; Mandible

\section{Introduction}

The inferior alveolar nerve may extend beyond the mental foramen in an anterior and inferior direction, curving back to the foramen and forming a loop, which has been termed the anterior loop of the inferior alveolar nerve, ${ }^{1-3}$ of the mental nerve, ${ }^{4-7}$ or of the mandibular

*This study was financially supported by the Fundação de Amparo à Ciência e Tecnologia do Estado de Pernambuco (FACEPE).

Received September 30, 2015; Revised January 13, 2016; Accepted January 26, 2016 *Correspondence to : Dr. Flávia Maria de Moraes Ramos-Perez

Universidade Federal de Pernambuco. Departamento de Clinica e Odontologia. Preventiva. Área de Radiologia Odontológica. $4^{\mathrm{a}}$ Travessa Professor Artur de Sá, s/n. Cidade Universitária. CEP 50740-521. Recife/PE, Brazil

Tel) 55-81-2126-8342, Fax) 55-81-2126-8817, E-mail) flavia_radio@hotmail.com canal. ${ }^{8,9}$ Although it is a benign anatomical variation, its accurate identification is essential for surgical planning, particularly for dental implant placement, ${ }^{1}$ in order to prevent iatrogenic complications and to ensure the effectiveness of surgical procedures performed in this region., ${ }^{4,8}$

Strikingly variable results regarding the prevalence of anterior loops of the inferior alveolar nerve have been reported in the literature, ranging from $22 \%-28 \%{ }^{6}$ to $88 \%$, and a maximum length of $11 \mathrm{~mm}$ has been reported. ${ }^{10}$ Differences in evaluation techniques (conventional radiographs, computed tomography [CT] scans, and direct anatomical analyses) may have influenced these discrepancies. Furthermore, the criteria for measuring the ante- 
rior loop should be considered, since some authors have used anatomical planes to identify the anterior limit of the mandibular canal, ${ }^{2,8,9,11}$ while others have differentiated between the anterior loop and the incisive canal by evaluating the diameters of these structures. ${ }^{1,3,12}$ In these cases, canals of at least $3 \mathrm{~mm}$ in diameter were considered to be part of the anterior loop. Thus, it is essential to use a diagnostic method that allows the satisfactory visualization and correct measurement of anatomical variations.

Cone-beam CT (CBCT) presents several advantages, such as allowing the three-dimensional (3D) assessment of craniofacial structures without distortion or overlapping images, in addition to a lower radiation dose than multislice CT. Moreover, CBCT is considered the standard method for assessing bone tissue $\mathrm{e}^{13,14}$ and is able to determine the presence and length of the anterior loop with precision and reliability. ${ }^{2,3}$

It should be highlighted that significant individual anatomical variations are present to various extents among different populations and ethnic groups. Thus, it is essential to conduct research in different populations. It is very important to precisely identify and to preserve neurovascular bundles in order to avoid sensorineural damage in the interforaminal region. The aim of this study was to use CBCT images to evaluate the prevalence and extent of the anterior loop of the mandibular canal in a Brazilian sample population.

\section{Materials and Methods}

This retrospective study was performed with the approval of the Research Ethics Committee of the Health Sciences Center, Federal University of Pernambuco, Brazil (protocol number 02158112.3.0000.5208).

From a pool of 1,500 consecutive CBCT scans of the jaws obtained between January 2010 and July 2010 in a private radiology clinic in Recife, Pernambuco, Brazil, 250 images of the mandibles were randomly selected, totaling 500 hemimandibles. CBCT images were included if they were of adequate diagnostic quality, without fractures and/or bone disease that compromised the region of the mental foramen. The exclusion criterion was the presence of implants or metal artifacts located in the foramen region.

The CBCT scans were acquired using an i-CAT Next Generation unit (Imaging Sciences International, Hatfield, PA, USA). The CBCT exposure parameters were $120 \mathrm{kV}, 3-8 \mathrm{~mA}, 0.25 \mathrm{~mm}$ voxel size, and $26 \mathrm{~s}$ acquisition time. Subsequently, the images were reconstructed using

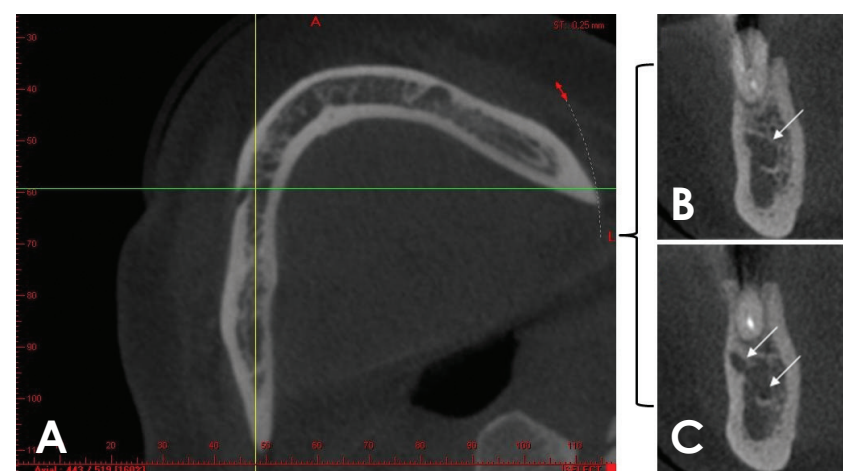

Fig. 1. Assessment of the anterior loop. A. Axial reconstruction rotated towards the side being examined, with the mandibular canal positioned parallel to the sagittal plane (vertical line). B and C. The coronal reconstructions obtained immediately after the most mesial point of the mental foramen show a single (arrow) and double (arrows) hypodense image, respectively.

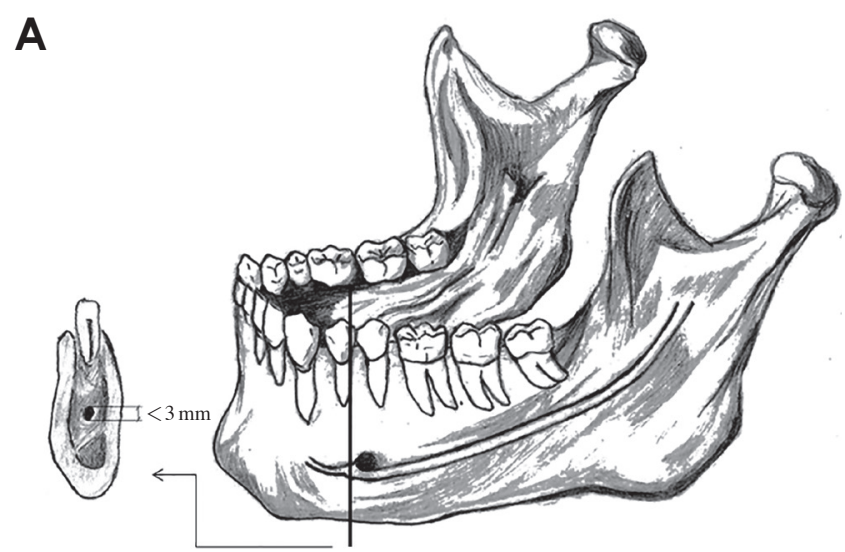

B

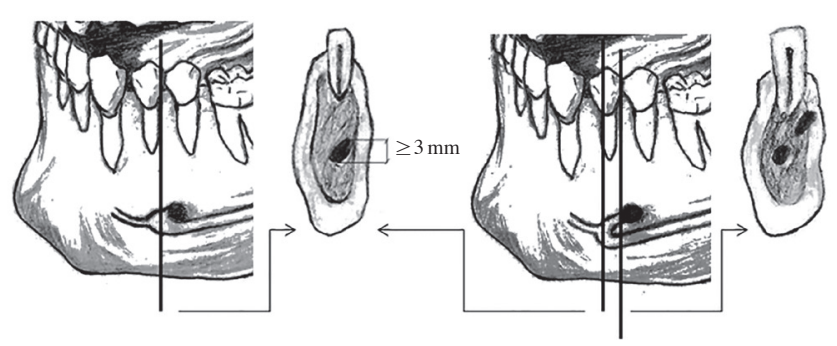

Fig. 2. The course of the mandibular canal, with the absence or presence of an anterior loop. A. The incisive canal and its corresponding image in the coronal reconstruction, with a diameter that must be less than $3 \mathrm{~mm}$. B. The anterior loop and its corresponding image in the coronal reconstruction, which, depending on the place it is obtained, can be represented by a round hypodense area with a diameter of at least $3 \mathrm{~mm}$ or by two round hypodense areas.

XORAN CAT version 2.0.21 (Xoran Technologies, Ann Arbor, MI, USA). Multiplanar reconstructions including axial, coronal, and sagittal images were obtained and evaluated concurrently by two trained and calibrated ob- 


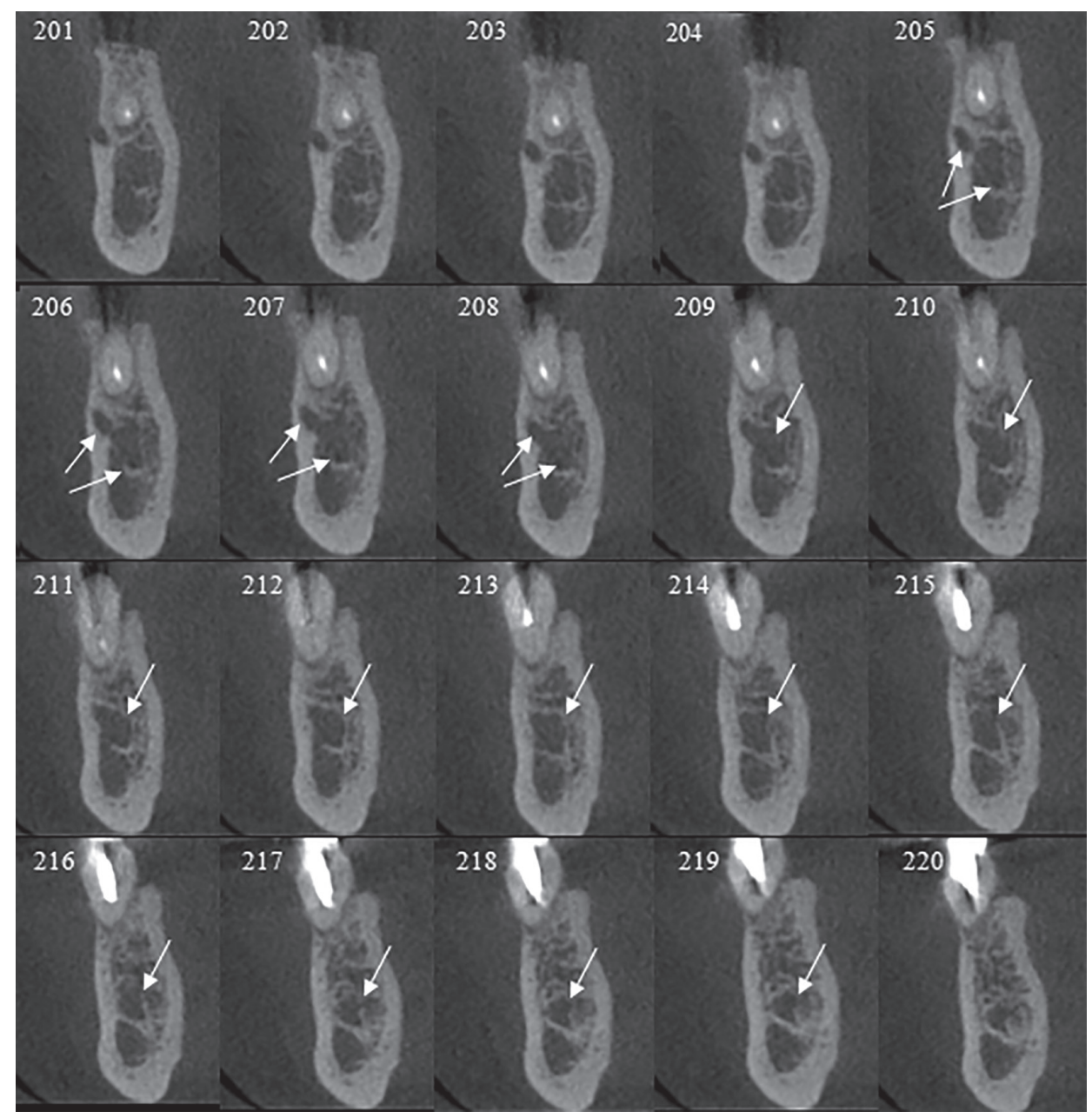

Fig. 3. In coronal reconstructions, the anterior loop can be seen as two round hypodense areas in images 205-208 and as a single round hypodense area (with a diameter of at least $3 \mathrm{~mm}$ ) in images 209-219. Measurement: $15 \times 0.25=3.75 \mathrm{~mm}$.

servers, in a darkened room, on a 19-inch liquid-crystal display screen (Infoway - Itautec, Taubaté, SP, Brazil). Furthermore, the density and contrast of the images were adjusted to assist the evaluators during identification and measurement procedures. Disagreements were discussed and a consensus was reached.

The axial reconstruction image was initially used. The volume was rotated towards the side being analyzed, in order to position the long axis of the mandibular canal parallel to the sagittal plane, and to position the coronal reconstruction perpendicular to the region of interest. The coronal reconstruction image was then used, and immediately afterwards, the most mesial point of the mental foramen was obtained in order to identify the presence or absence of an anterior loop. In the coronal sections, it was possible to identify two basic types of images: one with an anterior extension represented by a single round hypodense image and the other characterized by 2 round hypodense images (Fig. 1).

According to Apostolakis and Brown, ${ }^{1}$ the anterior loop can be differentiated from the incisive canal based on the fact that the incisive canal has a diameter of less than 3 $\mathrm{mm}$. When only a single round hypodense image was visualized, it was interpreted as the incisive canal if it exhibited a diameter smaller than $3 \mathrm{~mm}$. If the diameter was larger than $3 \mathrm{~mm}$, the anterior extension of the mandibular canal was considered to be an anterior loop. An anterior loop was also considered to be present when 2 round hypodense areas were observed, with one corresponding to the lumen of the mandibular canal that traverses the mental foramen anteriorly and inferiorly, and the other reflecting the doubling back (loop) of the mandibular canal, leading to the externalization of the inferior alveolar nerve. Figure 2 presents these possible representations of the anterior extension of the mandibular canal and the corresponding images in coronal reconstructions.

The length of the anterior loop was measured using the amount of consecutive coronal reconstructions situated between the anterior border of the mental foramen and the anterior border of the loop. This number was multiplied 


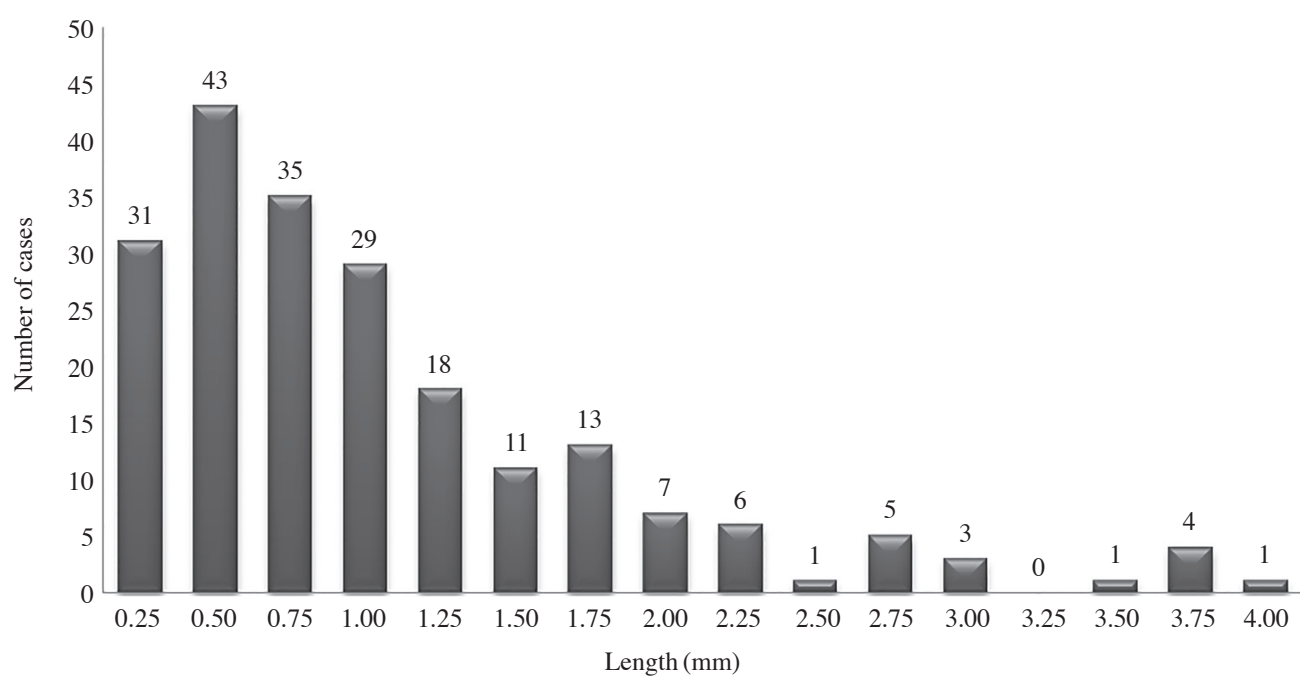

Fig. 4. The number of cases of anterior loop of the mandibular canal and their respective lengths.

by the thickness of the slices $(0.25 \mathrm{~mm})$.

When the anterior loop appeared as 2 round hypodense areas, its length was measured by counting the number of reconstructions in which the 2 round hypodense images appeared, added to the number of reconstructions in which the canal diameter was at least $3 \mathrm{~mm}$. The number of slices was then multiplied by the thickness of the reconstructions ( $0.25 \mathrm{~mm}$ ) (Fig. 3).

The data were summarized as absolute and relative frequencies. The Pearson chi-square test was used to evaluate the presence of an anterior loop with regard to age, gender, and the side of the hemimandible. The relationships of length with age, gender, and hemimandible side were evaluated using linear regression. P-values $<.05$ were considered to indicate statistical significance.

\section{Results}

The CBCT scans of 250 patients were evaluated, among whom $156(62.4 \%)$ were women and $94(37.6 \%)$ were men, with ages ranging between 13 and 87 years (mean age, $51.4 \pm 15.3$ years). The anterior loop was visualized in $208(41.6 \%)$ of the 500 hemimandibles analyzed, and the mean length was $1.1 \pm 0.8 \mathrm{~mm}$ (range, $0.25-4.00 \mathrm{~mm}$ ). These findings were analyzed according to gender, the side of the hemimandible (right and left), and the age of the patient (Table 1).

A significantly higher prevalence was found among men than among women $(\mathrm{p}=0.014)$. Furthermore, although the variability of the length of the anterior loop was greater in women, its mean length was greater in men.

The highest prevalence was found between the second and fifth decades of life, and the greatest variation in length was observed in the sixth decade. No significant differences were observed in length $(p=0.696)$ or prevalence $(\mathrm{p}=0.650)$ between the right and left sides.

Of the 208 hemimandibles in which an anterior loop was identified, in 138 cases $(66.3 \%)$ the length was less $1.00 \mathrm{~mm}$. In 49 cases $(23.6 \%)$, the length was between $1.25 \mathrm{~mm}$ and $2.00 \mathrm{~mm}$, in 15 cases $(7.2 \%)$ the length was between $2.25 \mathrm{~mm}$ and $3.00 \mathrm{~mm}$, and in only 6 cases $(2.9 \%)$ the length was between $3.25 \mathrm{~mm}$ and $4.00 \mathrm{~mm}$. Thus, the loop was $2 \mathrm{~mm}$ or shorter in approximately $90 \%$ of the cases. These findings are presented in Figure 4.

An anterior loop was visualized in 135 (54.0\%) of the 250 patients evaluated. Table 2 summarizes our findings according to the unilateral or bilateral presence of an anterior loop.

\section{Discussion}

The study of the anterior loop of the mandibular canal is a relatively new area of research, and most studies have been conducted in the last few years. ${ }^{1-3,6,7,12,15}$ The increased interest in the subject is directly related to the greater frequency of operations for the placement of dental implants. Several methods have been used to assess this type of anatomical repair, including panoramic radiography, ${ }^{16} \mathrm{CT},{ }^{12,15} \mathrm{CBCT},{ }^{1-3,6,7}$ anatomical measurements, ${ }^{8,10}$ and other related methods. ${ }^{5,9,11}$

The sample in this study was mainly composed of pati- 
Table 1. Distribution of cases of with and without an anterior loop of the mandibular canal and statistical analyses according to the side of the mouth and the gender and age of the patient.

\begin{tabular}{|c|c|c|c|c|c|c|}
\hline \multirow{2}{*}{ Group (n) } & \multirow{2}{*}{$\begin{array}{c}\text { Total } \\
\text { number }\end{array}$} & \multirow{2}{*}{$\begin{array}{c}\text { Absence of } \\
\text { anterior loop } \\
n(\%)\end{array}$} & \multirow{2}{*}{$\begin{array}{c}\text { Presence of } \\
\text { anterior loop } \\
\mathrm{n}(\%)\end{array}$} & \multicolumn{3}{|c|}{ Anterior loop } \\
\hline & & & & Range (mm) & Median & Mean \pm SD \\
\hline \multicolumn{7}{|l|}{ Gender } \\
\hline Male (94) & 188 & $96(51.1)$ & 92 (48.9) & $0.25-3.75$ & 1.00 & $1.22 \pm 0.92$ \\
\hline Female (156) & 312 & $196(62.8)$ & $116(37.2)$ & $0.25-4.00$ & 0.75 & $0.94 \pm 0.66$ \\
\hline \multicolumn{7}{|l|}{ Hemimandible } \\
\hline Right & 250 & $148(59.2)$ & $102(40.8)$ & $0.25-3.75$ & 0.75 & $1.06 \pm 0.83$ \\
\hline Left & 250 & 144 (57.6) & $106(42.4)$ & $0.25-4.00$ & 0.75 & $1.07 \pm 0.77$ \\
\hline \multicolumn{7}{|l|}{ Age (years) } \\
\hline $13-19(10)$ & 20 & $6(30.0)$ & $14(70.0)$ & $0.25-3.00$ & 1.25 & $1.32 \pm 0.89$ \\
\hline 20-29(17) & 34 & $15(44.1)$ & $19(55.9)$ & $0.25-1.75$ & 0.75 & $0.92 \pm 0.45$ \\
\hline $30-39(25)$ & 50 & $28(56.0)$ & $22(44.0)$ & $0.25-3.00$ & 1.00 & $1.25 \pm 0.86$ \\
\hline $40-49(46)$ & 92 & $49(53.3)$ & $43(46.7)$ & $0.25-2.75$ & 0.75 & $0.90 \pm 0.61$ \\
\hline $50-59(78)$ & 156 & $94(60.3)$ & $62(39.7)$ & $0.25-4.00$ & 1.00 & $1.24 \pm 0.99$ \\
\hline 60-69(49) & 98 & $67(68.4)$ & 31 (31.6) & $0.25-3.50$ & 0.75 & $0.81 \pm 0.64$ \\
\hline $70-79(20)$ & 40 & $27(67.5)$ & $13(32.5)$ & $0.25-2.25$ & 0.75 & $0.94 \pm 0.63$ \\
\hline $80-87(5)$ & 10 & $6(60.0)$ & $4(40.0)$ & $0.50-2.25$ & 1.25 & $1.31 \pm 0.94$ \\
\hline \multicolumn{7}{|l|}{ Total } \\
\hline Full sample & 500 & $292(58.4)$ & 208 (41.6) & $0.25-4.00$ & 0.75 & $1.06 \pm 0.80$ \\
\hline
\end{tabular}

SD: standard deviation.

Table 2. Results regarding the unilateral or bilateral presence of an anterior loop of the mandibular canal.

\begin{tabular}{lc}
\hline Anterior loop & $\mathrm{n}(\%)$ \\
\hline Bilateral & $73(54.1)$ \\
Unilateral: left & $33(24.4)$ \\
Unilateral: right & $29(21.5)$ \\
\hline All & $135(100)$ \\
\hline
\end{tabular}

ents aged between 50 and 60 years, as in the study carried out by Apostolakis and Brown ${ }^{1}$, and included a majority of females, similarly to other studies in the literature. . $^{1-3,5,6,10}$

Our study showed that the anterior loop of the mandibular canal was present in $41.6 \%$ of all cases. This is higher than the prevalence reported by Kuzmanovic et al., ${ }^{11}$ Kaya et al., ${ }^{5}$ and Ngeow et al., ${ }^{16}$ who conducted their studies using panoramic radiographic images. The relatively low prevalence rates reported in those studies may have reflected the failure of panoramic radiography to detect the presence of the anterior loop, due to the limitations and disadvantages of radiography, such as two-dimensionality, distortion, and the presence of overlapping structures. Moreover, panoramic radiography can overestimate or underestimate the presence and extent of the anterior loop, ${ }^{5,11}$ and is not considered a reliable modality for evaluating this anatomical variant. ${ }^{16}$
In contrast, studies using anatomical analysis ${ }^{8-10}$ or three-dimensional images ${ }^{1,3,5,7,12}$ have demonstrated higher rates of prevalence for the anterior loop, with values ranging from $34 \%{ }^{5}$ to $88 \% .{ }^{10}$ De Oliveira-Santos et al., ${ }^{6}$ even when using CBCT, observed a prevalence of $22 \%$ $28 \%$. However, this low value can be explained by the fact that those authors discarded loops smaller than $2 \mathrm{~mm}$ in length because they considered them clinically insignificant.

At least one anterior loop was visualized in over half of the patients (54.0\%) in our sample. Most of the anterior loops were observed bilaterally, followed by the left and right sides, respectively. These findings are similar to those reported by Apostolakis and Brown, ${ }^{1}$ who observed an anterior loop in approximately $57 \%$ of patients, mostly bilaterally. In the survey conducted by Filo et al., ${ }^{3}$ a loop was identified in $78.84 \%$ of patients, with the majority being bilateral, followed by being on the right and left sides, respectively.

The mean length of the anterior loops found in our study was $1.1 \mathrm{~mm}$, a value close to those that have been reported in the literature. ${ }^{1,3,15}$ The largest anterior loop observed was $4 \mathrm{~mm}$. Despite being clinically relevant in extent, ${ }^{6}$ this length was far lower than the maximum lengths of $9 \mathrm{~mm}$ reported by Uchida et al. ${ }^{9}$ and $11 \mathrm{~mm}$ reported by Neiva et al. ${ }^{10}$ Moreover, our research demonstrated that in $97.1 \%$ of cases, the length of the anterior loop was less 
than or equal to $3 \mathrm{~mm}$, similarly to the findings of Apostolakis and Brown ${ }^{1}$ and Filo et al. ${ }^{3}$ that approximately $95 \%$ of cases showed lengths of up to $3 \mathrm{~mm}$.

In our study, the anterior limit of the anterior loop was determined both based on the existence of two separate canals beyond the mental foramen and by using its diameter in all cross-sections. For this purpose, a minimum diameter of $3 \mathrm{~mm}$ was established, similar to the values used in other studies. ${ }^{1,3,12}$ In contrast, de Oliveira-Santos et al. ${ }^{6}$ and Kaya et al. ${ }^{5}$ did not measure the diameter of the canal and considered the anterior loop to be present only in cross-sections with 2 round hypodense images, with a structure that they referred to as "8-like." This method may have underestimated the real loop length.

We found that the anterior loop was larger and more prevalent in younger patients and male patients. No significant differences between the right and left sides were found. Other studies have shown the length of the anterior loop to be significantly related to male gender, ${ }^{1,2,8,9,12}$ the right side, ${ }^{1}$ and to the sixth decade of life. ${ }^{8}$ Some authors, however, have found no significant relationships between these variables (age, gender, and side) and length $\mathrm{th}^{3,6}$ or prevalence $^{3,6,12}$ of anterior loops.

Although anatomical studies on cadavers provide important and accurate information about the prevalence and variation in length of the anterior loop, CT is the method that actually corresponds to clinical practice and adequately satisfies pre-surgical planning requirements. Furthermore, CT images have been proven to exhibit high precision and reliability in the diagnosis of the anterior loop ${ }^{2,9}$ and other anatomical landmarks in the mandible. ${ }^{6}$

As previously stated, CT images are very effective exams for the diagnosis of anterior loops of the mandibular canal and can be used with great reliability to identify their presence and measure their length., ${ }^{2,3}$ However, CBCT is preferable and is most often used in dental practice due to its advantages, including a lower cost and a lower radiation dose, in combination with the fact that CBCT image quality is comparable or even superior to that of multislice CT for evaluating dentomaxillofacial structures. $^{13,14}$

The high prevalence and significant extent of the anterior loop found in this study highlight the importance of knowledge regarding this anatomical variation. For this reason, it is necessary for professionals to identify the presence of anterior loops and to measure them correctly when planning procedures involving the interforaminal region.

In conclusion, there was high prevalence of anterior loop of the mandibular canal, with the maximum length of $4.00 \mathrm{~mm}$. However, in spite of the wide variation in length, the majority of loops were less than $1 \mathrm{~mm}$ long.

\section{References}

1. Apostolakis D, Brown JE. The anterior loop of the inferior alveolar nerve: prevalence, measurement of its length and a recommendation for interforaminal implant installation based on cone beam CT imaging. Clin Oral Implants Res 2012; 23 : 1022-30.

2. Rosa MB, Sotto-Maior BS, Machado Vde C, Francischone CE. Retrospective study of the anterior loop of the inferior alveolar nerve and the incisive canal using cone beam computed tomography. Int J Oral Maxillofac Implants 2013; 28: 388-92.

3. Filo K, Schneider T, Locher MC, Kruse AL, Lübbers H. The inferior alveolar nerve's loop at the mental foramen and its implications for surgery. J Am Dent Assoc 2014; 145: 260-9.

4. Greenstein G, Tarnow D. The mental foramen and nerve: clinical and anatomical factors related to dental implant placement: a literature review. J Periodontol 2006; 77: 1933-43.

5. Kaya Y, Sencimen M, Sahin S, Okcu KM, Dogan N, Bahcecitapar M. Retrospective radiographic evaluation of the anterior loop of the mental nerve: comparison between panoramic radiography and spiral computerized tomography. Int J Oral Maxillofac Implants 2008; 23: 919-25.

6. de Oliveira-Santos C, Souza PH, de Azambuja Berti-Couto S, Stinkens L, Moyaert K, Rubira-Bullen IR, et al. Assessment of variations of the mandibular canal through cone beam computed tomography. Clin Oral Investig 2012; 16: 387-93.

7. Parnia F, Moslehifard E, Hafezeqoran A, Mahboub F, Mojaver-Kahnamoui H. Characteristics of anatomical landmarks in the mandibular interforaminal region: a cone-beam computed tomography study. Med Oral Patol Oral Cir Bucal 2012; 17: e420-5.

8. Uchida Y, Yamashita Y, Goto M, Hanihara T. Measurement of anterior loop length for the mandibular canal and diameter of the mandibular incisive canal to avoid nerve damage when installing endosseous implants in the interforaminal region. $\mathbf{J}$ Oral Maxillofac Surg 2007; 65: 1772-9.

9. Uchida Y, Noguchi N, Goto M, Yamashita Y, Hanihara T, Takamori H, et al. Measurement of anterior loop length for the mandibular canal and diameter of the mandibular incisive canal to avoid nerve damage when installing endosseous implants in the interforaminal region: a second attempt introducing cone beam computed tomography. J Oral Maxillofac Surg 2009; 67: 744-50.

10. Neiva RF, Gapski R, Wang HL. Morphometric analysis of implant-related anatomy in Caucasian skulls. J Periodontol 2004; 75: 1061-7.

11. Kuzmanovic DV, Payne AG, Kieser JA, Dias GJ. Anterior loop of the mental nerve: a morphological and radiographic study. Clin Oral Implants Res 2003; 14: 464-71.

12. Li X, Jin ZK, Zhao H, Yang K, Duan JM, Wang WJ. The prevalence, length and position of the anterior loop of the inferior alveolar nerve in Chinese, assessed by spiral computed tomography. Surg Radiol Anat 2013; 35: 823-30. 
13. Liang X, Jacobs R, Hassan B, Li L, Pauwels R, Corpas L, et al. A comparative evaluation of cone beam computed tomography (CBCT) and multi-slice CT (MSCT) Part I. On subjective image quality. Eur J Radiol 2010; 75: 265-9.

14. Suomalainen A, Kiljunen T, Käser Y, Peltola J, Kortesniemi M. Dosimetry and image quality of four dental cone beam computed tomography scanners compared with multislice computed tomography scanners. Dentomaxillofac Radiol 2009; 38:
367-78.

15. Watanabe H, Mohammad Abdul M, Kurabayashi T, Aoki H. Mandible size and morphology determined with CT on a premise of dental implant operation. Surg Radiol Anat 2010; 32: 343-9.

16. Ngeow WC, Dionysius DD, Ishak H, Nambiar P. A radiographic study on the visualization of the anterior loop in dentate subjects of different age groups. J Oral Sci 2009; 51: 231-7. 